\title{
Geometry-based Localization for GPS Outage in Vehicular Cyber Physical Systems
}

\author{
Omprakash Kaiwartya, Member IEEE, Yue Cao, Member IEEE, Jaime Lloret, Senior Member IEEE, Sushil Kumar, \\ IEEE Member, Nauman Aslam, Member IEEE, Rupak Kharel, Member IEEE, Abdul Hanan Abdullah, Member
}

\author{
IEEE, Rajiv Ratn Shah, Member IEEE
}

\begin{abstract}
Vehicular localization has witnessed significant attention due to the growing number of location-based services in vehicular cyber physical systems (VCPS). In vehicular localization, GPS outage is a challenging issue considering the growing urbanization including high rise buildings, multi-level flyovers and bridges. GPS-free and GPS-assisted cooperative localization techniques have been suggested in literature for GPS outage. Due to the cost of infrastructure in GPS-free techniques, and the absence of location aware neighbors in cooperative techniques, efficient and scalable localization is a challenging task in VCPS. In this context, this paper proposes a geometry-based localization for GPS outage in VCPS (GeoLV). It is a GPSassisted localization which reduces location aware neighbor constraint of cooperative localization. GeoLV utilizes mathematical geometry to estimate vehicle location focusing on vehicular dynamics and road trajectory. The static and dynamic relocations are performed to reduce the impact of GPS outage on location-based services. A case study based comparative performance evaluation has been carried out to assess the efficiency and scalability of GeoLV. It is evident from the results that GeoLV handles both shorter and longer GPS outage problem better than the state-of-the-art techniques in VCPS.

Index Terms - Vehicular cyber physical system, GPS outage, Vehicular communication, Vehicular localization
\end{abstract}

\section{INTRODUCTION}

$\mathrm{D}$ riverless car is one of the best examples of the significance of location information in vehicular cyber physical systems (VCPS) environments (see Fig. 1) [1, 2]. The other examples of location-based services in intelligent transportation system attest the worthiness of location information [3]. The services include cooperative vehicular communication for safety, navigation, emergency response and driver assistance [4, 5]. Recently, vehicular localization has witnessed significant attention due to the growing number of location-based services in VCPS [6].

The research is supported by the gLINK project at the Northumbria University UK (EU grant No: 2014-0861/001-001- EM Action 2- Partnerships).

O. Kaiwartya, Y. Cao (Corresponding Author), N. Aslam are with the Northumbria University, Newcastle, NE1 8ST, UK. Email: omprakash.kaiwartya; yue.cao; numan.aslam@northumbria.ac.uk

J. Lloret is with the Universitat Politecnica de Valencia, 46022, Valencia, Spain, Email: jlloret@dcom.upv.es

S. Kumar is with the Jawaharlal Nehru University, New Delhi, 110067 , India, Email: skdohare@mail.jnu.ac.in

R. Kharel is with the School of Engineering, Manchester Metropolitan University, M1 5GD, UK Email: r.kharel@mmu.ac.uk

A.H. Abdullah is with the Universiti Teknologi Malaysia (UTM), Johor Bahru, 81310, Malaysia. Email: hanan@utm.my

R. R. Shah is with the School of Information Systems, Singapore Multimedia University, Singapore, 178902. Email: rajivshah@ @mu.edu.sg

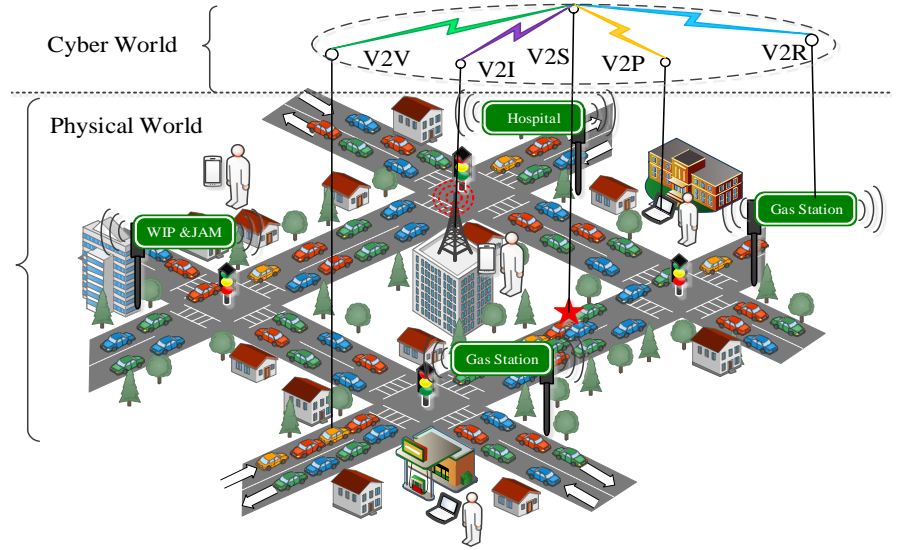

Fig. 1. The cyber and physical world in VCPS environments, Vehicle-toVehicle (V2V), Vehicle-to-Infrastructure (V2I), Vehicle-to-Personal devices (V2P), Vehicle-to-Roadside (V2R), Vehicle-to-Sensors (V2S) communication

Initially, GPS-based localization techniques were developed for VCPS environments, due to the widespread adaptation of GPS for localization considering simplicity and cost effective solution. Various location based services have been developed using GPS-based localization for safety and efficiency in transportation [7-9]. GPS-based location of vehicles has been utilized for measuring vehicle density on roads [7], predicting collision at junctions [8], and determining roadside unite (RSU) location [9]. GPS-based localization implicitly assumes non-disruptive availability of GPS signal with sufficient quality, from at least two satellites throughout the VCPS environments [10]. Considerable performance degradation in GPS-based localization has been observed in case of GPSoutage $^{1}$ in VCPS environments with high rise buildings, multilevel flyovers and different types of road trajectories [16]. This has resulted as the development of infrastructure-based GPS-free localization techniques [11-15].

GPS-free localization includes received signal strength indicator (RSSI) based localization [11], radio frequency identification (RFID) based localization [12, 13], and RSU based localization $[14,15]$. These GPS-free localizations are based on either radio ranging techniques including RSSI, and Time of Arrival (TOA), or RFID techniques. However, the performance of radio ranging techniques significantly degrades due to the mobility in VCPS environments, particularly with high rise buildings and multi-level flyovers [16]. The deployment of RFID tags on roads or RSU alongside roads significantly increases the cost of infrastructure for localization [17]. The concerns over the technical feasibility of infrastructure deployment, and the rising cost of GPS-free 
localization, push the new way of solution for GPS outage problem.

Recently, GPS-assisted cooperative localization has received significant attention as cost-effective and feasible solution for GPS outage problem [18-23]. In cooperative localization, vehicles utilize GPS in case of availability of signal. Cooperative communication among neighboring vehicles as present knowledge, and last GPS data as past knowledge, are utilized in case of GPS outage for shorter or longer duration. Shorter outage occurs on single road section, whereas longer outage involves multiple curved or angular road sections. It includes grid based localization using cooperative neighbors (two or more) $[18,19]$, Doppler shift based localization [20], GPS pseudo range based localization [21], and data fusion using Kalman filter based localization $[22,23]$. However, the presence of two or more location aware neighbor is the constraint of grid based localizations. The undesirable aspects of range rating technique include the performance degradation in the presence of radio obstacles, and the minimum speed difference requirement for observable Doppler shift. The limitation of pseudo range based localization is the assumption of availability of GPS pseudo range from four common satellites. The assumption of relative positioning capability with neighboring vehicles is the constraint of data fusion based localizations. The impact of GPS outage on location-based services is not considered in the aforementioned localization techniques.

In summary, most of the GPS-assisted localization techniques have assumed the availability of sufficient number of (two or more) location aware neighboring vehicles for location fusion [18, 19]. The minimum speed difference between the target vehicle and its neighboring vehicles has been assumed, which is required to generate observable Doppler shift [20]. We argue both the assumptions including GPS aware neighboring vehicles, and the minimum speed difference are infeasible. Firstly, if a vehicle faces GPS outage on specific VCPS environments, how its neighboring vehicles in the same environments could know their GPS location. Therefore, if the target vehicle faces GPS outage, its neighboring vehicles would also face GPS outage. To this end, a localization technique addressing GPS outage problem must be free from the requirement of location aware neighbors. Secondly, the minimum speed difference could not be guaranteed in urban environments, considering higher traffic density and speed limitation rules. It reduces the applicability of minimum speed difference based range rating techniques for localization in urban VCPS environments. It is worth noting that the efficient relocation ${ }^{2}$ reduces the impact of GPS outage on location-based services.

In this context, this paper proposes geometry-based localization for GPS outage in VCPS environments (GeoLV). It is a GPS-assisted localization which reduces the two assumptions of cooperative localization. The assumptions include location aware neighboring vehicles, and the minimum speed difference between the target vehicle and its neighboring vehicles. Compared to literature on GPS-assisted localization, our novelty focuses on geometry-based localization by utilizing vehicular dynamics, road trajectories. It neither requires location aware neighboring vehicles, nor the minimum speed difference between the target and neighboring vehicles. Specifically, we aim to answer the following four questions:

1) How to convert shorter GPS outage problem on straight road section into a geometrical problem, and how it can be solved using vehicular dynamics?

2) How to convert longer GPS outage problem on curved and angular road sections into a geometrical problem, and how it can be solved using road trajectory along with vehicular dynamics?

3) How to reduce the impact of GPS outage on location based services in VCPS environments using static and dynamic relocations?

4) Is the provisioned geometry-based localization efficient and scalable, considering the impact of GPS outage duration and vehicle density on the performance of GeoLV?

The rest of the article is organized into following sections. Section II reviews related literature on localization in VCPS focusing on GPS-based, GPS-free, and GPS-assisted localizations. Section III presents the detail of the proposed GeoLV including the conversion of GPS outage problems into geometrical problems with their solution. Section IV discusses analytical and case study based comparative performance evaluation, followed by conclusion made in section $\mathrm{V}$.

\section{RELATED WORK}

\section{A. GPS-based Localization}

GPS-based localization has been utilized to track vehicles and measure the congestion based on the current speed of traffic on roads [7]. A route planning service initially suggests better route for the termed destination by comparing the traffic speed on each route, before the start of the journey. The initial route suggestion has been updated using GPS-based localization aided real time traffic information. The complexity analysis and mathematical validation of the route planner have not been provided. GPS based localization has been enhanced to cope up in urban canyon vehicular environments [10]. The minimum number of GPS satellite requirement for localization have been reduced from four to two satellites. An extended Kalman filter with indirect solution approach has been utilized to solve the path resembling problem for a vehicle, from the pieces of curve of road network. The constraint in terms of GPS signal from at least two satellites reduces the applicability of the localization. The performance of inter-vehicle communication aided by GPS-based localization has been tested and analyzed through a collision predictor system [8]. Collisions at junction have been avoided by deviating from the trajectories which intersect at junctions. GPS outage problem has not been considered in this collision predictor system. GPS-based location of mobile vehicle and RSSI have been utilized to measure the location of RSUs [9]. A mobile vehicle has been used to collect RSSI of RSUs, and tag the information with own location before uploading to a server. The server has been used by vehicles to query location. In most of the works on GPS-based localization, either the GPS outage problem is not considered at all [7-9] or partially considered [10]. 


\section{B. GPS-free Localization}

RFID based localization has been suggested considering RFID tags on roads and RFID readers with vehicles [12]. Specifically, a new design of RFID reader for vehicular environment has been explored focusing on adaptive scheduling of read attempt. RFID tag deployment strategy has been suggested by deriving the tag deployment distance between two consecutive tags for junctions and continuous roads. RFID based localization has been improved using a kinematics integration algorithm [13]. It enables the estimation of location of vehicles without RFID coverage. Thus, the requirement of number of RFID tags on roads has been reduced. More precisely, in case of a vehicle on a tag on road, the tag's location has been considered as the location of the vehicle. The last tag's location and vehicle's kinematics have been utilized to estimate the location of a vehicle, which is out of range of any tag. However, the cost of two types of infrastructure, e.g., RFID tags on roads, and RFID reader with vehicles, reduces the feasibility of the localization.

For reducing the infrastructure cost of RFID-based localization from two types of infrastructure to one type infrastructure (RFID tags on roads), RSU based localization has been suggested using two-way reciprocal Time of Arrival (TOA) ranging technique [14]. Two sets of beacon messages from a pair of RSUs have been utilized by a vehicle to calculate distance from the pair of RSUs. The distance is converted into the location of vehicle using the known location of RSUs. An enhancement in RSU based localization has been suggested by reducing the number of RSU requirement from two RSUs to one RSU [15]. Kalman filter has been utilized to enhance the estimated location by fusing range based distance and vehicle's kinematics information. However, the deployment and maintenance of RSUs alongside roads is a challenging task considering the feasibility and cost aspects. The GPS-free localization techniques are based on either radio ranging techniques including RSSI and TOA, or RFID technique. The performance of radio ranging techniques significantly degrades in VCPS environments [16]. Moreover, the deployment of RFID tags on roads and attaching RFID readers with vehicles increase the infrastructure cost of localization, as compared to the usage of GPS with enhancement techniques [17].

\section{GPS-assisted Localization}

Grid based localization has been suggested for detection and tracking of vehicles [18]. It has been assumed that vehicles are equipped with laser scanner, short range radar and odometer. A multiple hypothesis tracker integrated with interacting model filter has been utilized for tracking on-themove vehicles. An enhancement in grid based localization (GOT) has been suggested with the help of three location aware neighboring vehicles [19]. It reduces the equipment requirement considered in [18]. The localization is based on the four different geographical patterns of three location aware neighboring vehicles. A location estimation technique has been derived using only addition operation. Thus, reduction in error propagation has been claimed from exponential to linear. The constraint in terms of the presence of three location aware neighboring vehicles reduces the applicability of grid based localization. The constraint of grid based localization has been reduced using Doppler shift [20]. A range rating technique has been utilized to enhance location information, without concerning on the availability of exact number of location aware neighbors. Doppler shift of the received signals from neighboring vehicles has been considered at the target vehicle. The limitation of range rating technique based localization is the requirement of minimum speed difference, for the observable Doppler shift in received signals.

For addressing the limitation of range rating technique based localization, tight integration based localization has been explored utilizing the concept of differential GPS (DGPS) [21]. The error in low level GPS data, i.e., pseudo range, has been corrected by obtaining double differences of observed pseudo ranges. Kalman filter has been utilized as the process and observation models in relative positioning of neighboring vehicles. Although the tight integration does not depend on radio ranging and range rating techniques yet, the constraint in terms of signal from two common GPS satellites reduces the applicability of the localization in urban vehicular environments. Another GPS-assisted localization using Kalman filter has been suggested focusing on inter vehicle communication (IVCAL) [22]. Relative distance from neighbors obtained via vehicular communication has been utilized to reduce the impact of line-of-sight and multipath related issues in localization. The location enhancement has been performed using Kalman filter based data fusion. The data fusion in cooperative localization has been improved using split covariance intersection filter, to reduce the consequences of inter-estimate correlation [23]. The undesirable aspect of data fusion based localization is the assumption of relative positioning capability with neighboring vehicles, or in other words location aware neighboring vehicles. In case of correlated successive positioning estimates, the effectiveness of data fusion using Kalman filter significantly reduces due to the error propagation.

\section{GeOMETRY BASED LoCALIZATION FOR GPS OUTAGE}

In this section, the design of the proposed GPS-assisted localization is presented in detail. It is assumed that each vehicle can measure speed, direction and time with sufficient accuracy. In GeoLV, a vehicle utilizes geometrical-modelbased estimation to derive its current location in case of GPS outage. Specifically, a vehicle $v_{i}$ estimates its current location $\left(x_{i}, y_{i}\right)$ using the present and past knowledge of vehicular dynamics and road trajectories. The present knowledge includes current direction $d_{i}$, speed $s_{i}$, and time stamp $t_{i}$ of the vehicle. The past knowledge includes the last known GPS location $\left(x_{i}^{\prime}, y_{i}^{\prime}\right)$, direction $d_{i}^{\prime}$, speed $s_{i}^{\prime}$, and time stamp $t_{i}^{\prime}$ of the vehicle. The location estimation is carried out considering the following three cases with straight, curved and angular road scenarios, however, the combination of three or more road sections is also possible.

- In the first case, shorter GPS outage is considered. The last known GPS location and the current location (to be estimated) are considered on a straight road section, either on the same direction lane or on the opposite direction lane. The present and past directions of the vehicle are either equal or opposite, i.e., $d_{i} \cong d_{i}^{\prime}$ or $\left|d_{i}-d_{i}^{\prime}\right| \cong 180^{\circ}$. 
- In the second case, longer GPS outage is considered. The last known GPS location and the current location of the vehicle is considered on two different road sections, with curved shape joining point. Any two road sections joining at any angle other than $0^{\circ}$ or $180^{\circ}$ are considered different road sections. The present and past directions of the vehicle are different (other than opposite), i.e., $d_{i} \neq d_{i}^{\prime}$ and $\left|d_{i}-d_{i}^{\prime}\right| \neq 180^{\circ}$.

- The third case also consider longer GPS outage, but with multiple and angular road sections, i.e., $d_{i} \perp d_{i}^{\prime}$ or $d_{i} \cong d_{i}^{\prime}$ after passing perpendicular road sections.

Table 1. Nomenclature

\begin{tabular}{ll}
\hline Notation & Description \\
\hline$v_{i}$ & $i^{t h}$ vehicle of the network \\
$\left(x_{i}, y_{i}\right)$ & Current location of $v_{i}$ \\
$\left(x_{i}^{\prime}, y_{i}^{\prime}\right)$ & Last GPS location of $v_{i}$ \\
$d_{i}$ & Direction of $v_{i}$ at $\left(x_{i}, y_{i}\right)$ \\
$d_{i}^{\prime}$ & Direction of $v_{i}$ at $\left(x_{i}^{\prime}, y_{i}^{\prime}\right)$ \\
$s_{i}$ & Speed of $v_{i}$ at $\left(x_{i}, y_{i}\right)$ \\
$s_{i}^{\prime}$ & Speed of $v_{i}$ at $\left(x_{i}^{\prime}, y_{i}^{\prime}\right)$ \\
$t_{i}$ & Time stamp at $\left(x_{i}, y_{i}\right)$ \\
$t_{i}^{\prime}$ & Time stamp at $\left(x_{i}^{\prime}, y_{i}^{\prime}\right)$ \\
$D$ & Distance between $\left(x_{i}, y_{i}\right)$ and $\left(x_{i}^{\prime}, y_{i}^{\prime}\right)$ \\
$D_{i}$ & Travel distance on $i^{t h}$ road section \\
$C_{i}$ & $i^{t h}$ circle considered for solving geometrical problems \\
$l_{i}$ & $i^{t h}$ line considered for solving geometrical problems \\
$\theta$ & Sector angle at $C_{3}$ \\
$\theta_{i}$ & $i^{t h}$ angle considered for solving geometrical problems \\
$r$ & Radius of curved road trajectory \\
$D_{H}$ & Present heading distance \\
$D_{H}^{\prime}$ & Past heading distance \\
$N_{r s}$ & Number of road sections \\
$E_{l t}$ & Expected lifetime of static relocation \\
$s_{r}$ & Relative speed \\
$d_{r}$ & Relative direction \\
$\mathcal{W}_{d_{i}}$ & Weight of direction of $i^{t h}$ neighbour \\
$\mathcal{W}_{s_{i}}$ & Weight of speed of $i^{t h}$ neighbour \\
$\mathcal{W}_{N_{d_{i}}}$ & Weight of number of direction change of $i^{\text {th }}$ neighbour \\
$\mathcal{W}_{N_{s_{i}}}$ & Weight of number of speed change of $i^{t h}$ neighbour \\
$\mathbb{w}_{D R}$ & Cumulative weight for dynamic relocation \\
\hline &
\end{tabular}

\section{A. Case I: Location Estimation on Straight Road Section}

In the first case, i.e., $d_{i} \cong d_{i}^{\prime}$ or $\left|d_{i}-d_{i}^{\prime}\right| \cong 180^{\circ}$, a vehicle receives GPS signal while moving on a straight road section. After a short period, the vehicle faces GPS outage problem on the same road section as depicted in Fig. 2. In this situation, the problem of estimation of present location on a straight road section is converted into a geometrical problem as depicted in Fig. 3. The problem can be geometrically stated as finding the location of a vehicle on a road section, which is $D$ distance away from the known location on the same road section. In this geometrical problem, $\left(x_{i}, y_{i}\right)$ and $\left(x_{i}^{\prime}, y_{i}^{\prime}\right)$ represent the unknown present and known past locations, $d_{i}$ and $d_{i}^{\prime}$ represent the present and past directions, $s_{i}$ and $s_{i}^{\prime}$ represent present and past speeds, $t_{i}$ and $t_{i}^{\prime}$ represent the current and the last time stamp (GPS availability time), and $D$ represents the distance between $\left(x_{i}, y_{i}\right)$ and $\left(x_{i}^{\prime}, y_{i}^{\prime}\right)$. The distance $D$ travelled by the vehicle since the last known location in the same or opposite direction can be calculated using average speed and travel time as expressed by Eq. (1).

$$
D=\left\{\begin{array}{c}
\left(t_{i}-t_{i}^{\prime}\right) \times \frac{\left(s_{i}+s_{i}^{\prime}\right)}{2}, d_{i} \cong d_{i}^{\prime} \\
\left|\left(\frac{\left(t_{i}-t_{i}^{\prime}\right)}{2} \times s_{i}\right)-\left(\frac{\left(t_{i}-t_{i}^{\prime}\right)}{2} \times s_{i}^{\prime}\right)\right|,\left|d_{i}-d_{i}^{\prime}\right| \cong 180^{\circ}
\end{array}\right.
$$

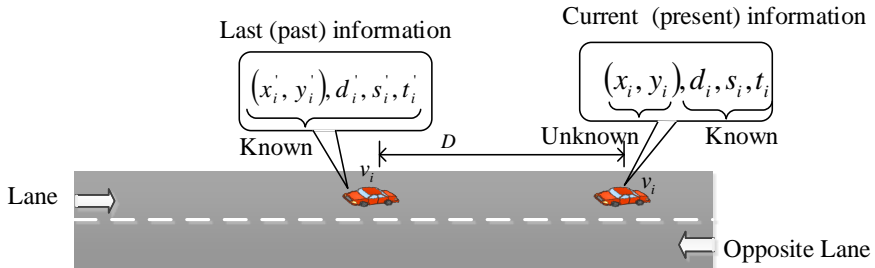

Fig. 2. GPS outage problem on a straight road section

The GPS outage problem on a straight road section can be solved using geometrical derivation of circle and line. To this end, a circle $C_{1}$ of radius $D$ with past location $\left(x_{i}^{\prime}, y_{i}^{\prime}\right)$ as center is considered, to convert the GPS outage problem into a geometrical problem as depicted in Fig. 3. In terms of geometry, the problem is to find the intersection point $\left(x_{i}, y_{i}\right)$ between the circle $C_{1}$ and line $l_{1}$, given the information related to vehicular dynamics. The center of the circle $\left(x_{i}^{\prime}, y_{i}^{\prime}\right)$ is a known location, and thus, the point $\left(x_{i}^{\prime}, y_{i}^{\prime}\right)$ on line $l_{1}$ is also known. Therefore, equation of circle $C_{1}$ and line $l_{1}$ can be expressed as given by Eq. (2) and (3), respectively.

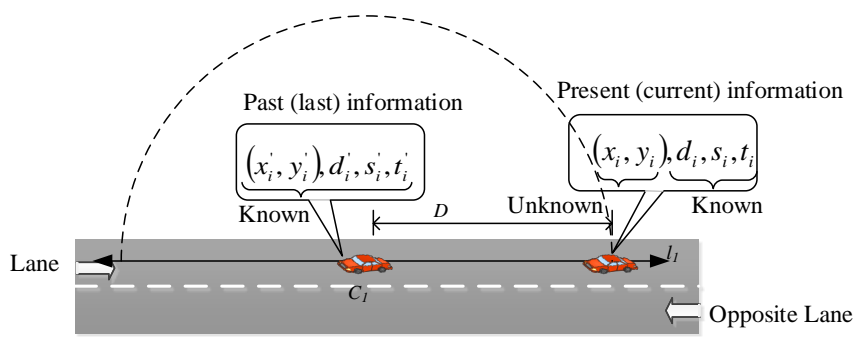

Fig. 3. Geometrical problem on a straight road section

$$
\left(x-x_{i}^{\prime}\right)^{2}+\left(y-y_{i}^{\prime}\right)^{2}=D^{2}
$$

and, $\quad\left(y-y_{i}^{\prime}\right)=d_{i}^{\prime}\left(x-x_{i}^{\prime}\right)$

The current unknown location $\left(x_{i}, y_{i}\right)$ is a point on the circle $C_{1}$ and line $l_{1}$. Therefore, the point satisfies Eq. (2) and (3). This can be expressed as given in Eq. (4) and (5).

$$
\begin{array}{ll} 
& \left(x_{i}-x_{i}^{\prime}\right)^{2}+\left(y_{i}-y_{i}^{\prime}\right)^{2}=D^{2} \\
\text { and, } \quad & \left(y_{i}-y_{i}^{\prime}\right)=d_{i}^{\prime}\left(x_{i}-x_{i}^{\prime}\right)
\end{array}
$$

By using Eq. (5), Eq. (4) can be simplified into two forms of quadratic equations as expressed by Eq. (6) and (7).

$$
\begin{aligned}
x_{i}^{2}-2 x_{i} x_{i}^{\prime}+\left(x_{i}^{\prime}\right)^{2}-\frac{D^{2}}{1+\left(d_{i}^{\prime}\right)^{2}}=0 \\
\text { and, } \quad y_{i}^{2}-2 y_{i} y_{i}^{\prime}+\left(y_{i}^{\prime}\right)^{2}-\frac{D^{2}\left(d_{i}^{\prime}\right)^{2}}{1+\left(d_{i}^{\prime}\right)^{2}}=0
\end{aligned}
$$

The two quadratic equations are solved to obtain the current location of vehicle $v_{i}$ as expressed by Eq. (8).

$$
\left(x_{i}, y_{i}\right)=\left(x_{i}^{\prime} \pm \frac{D}{\sqrt{\left(1+d_{i}^{\prime 2}\right)}}, y_{i}^{\prime} \pm \frac{D \times d_{i}^{\prime}}{\sqrt{\left(1+d_{i}^{\prime 2}\right)}}\right)
$$

\section{B. Case II: Location Estimation on Curved Road Sections}

In the second case, $d_{i} \neq d_{i}^{\prime}$ and $\left|d_{i}-d_{i}^{\prime}\right| \neq 180^{\circ}$, a vehicle $v_{i}$ receives GPS signal while moving on a road section. After a period, when the vehicle changes its direction and enters on 
a curved road section, the vehicle faces GPS outage problem on the curved road section as depicted in Fig. 4. In this situation, the problem of estimation of present location on double curved road sections, is converted into a geometrical problem as depicted in Fig. 5.

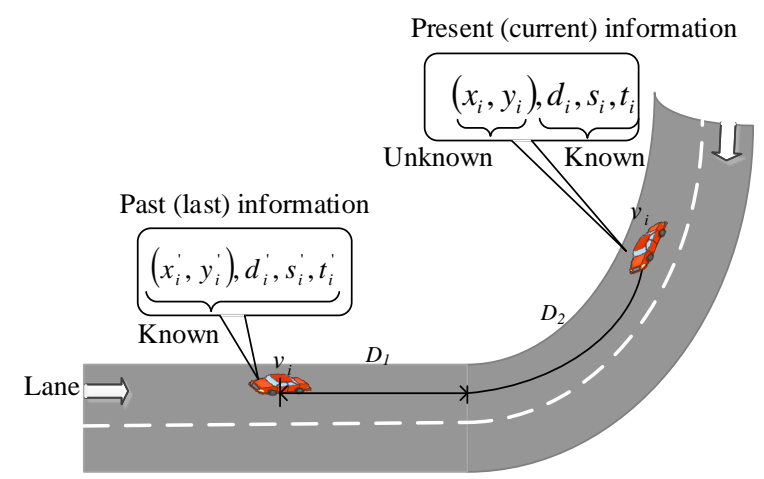

Fig. 4. GPS outage problem on double curved road sections

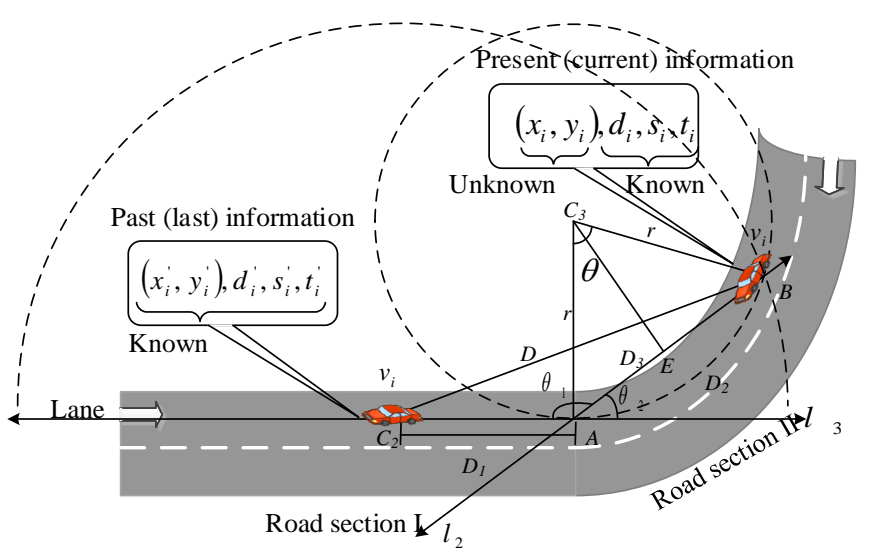

Fig. 5. Geometrical problem on double curved road sections

In this geometrical problem, $D_{1}=\frac{\left(t_{i}-t_{i}^{\prime}\right)}{2} \times s_{i}^{\prime}$ and $D_{2}=$ $\frac{\left(t_{i}-t_{i}^{\prime}\right)}{2} \times s_{i}$ represent the distance traveled by $v_{i}$ on first and second road section considering average travel time and speed. The GPS outage problem on double curved road sections can be solved using geometrical derivation of two circles and lines. To this end, two circles $C_{2}$ and $C_{3}$, and lines $l_{2}$ and $l_{3}$ are considered.

In terms of geometry, the problem is to find the intersection point $\left(x_{i}, y_{i}\right)$ between the circle $C_{2}$ and line $l_{2}$. Another line $l_{3}$ is considered which passes through the center of circle $C_{2}$ and intersects line $l_{2}$ with an angle $\theta_{2}$. The center of the circle $\left(x_{i}^{\prime}, y_{i}^{\prime}\right)$ is a known location, and thus, a point $\left(x_{i}^{\prime}, y_{i}^{\prime}\right)$ on the line $l_{3}$ is also known. The angle $\theta=\frac{D_{2}}{r}$ can be obtained using the radius $r$ of circle $C_{3}$, which is known from road trajectory, and circular arc distance $D_{2}$. The length of the cord $A B=$ $D_{3}=2 r \sin \left(\frac{\theta}{2}\right)$ can be derived considering right angle triangle $\triangle B E C_{3}$. The angle $C_{2} A B=\theta_{1}=180^{\circ}-\theta_{2}$ can be obtained using the known angle $\theta_{2}$ which is the current direction $d_{i}$ of vehicle $v_{i}$. The distance $D$ between the last known location $\left(x_{i}^{\prime}, y_{i}^{\prime}\right)$ and the current location $\left(x_{i}, y_{i}\right)$ of the vehicle can be derived by utilizing triangular geometry on triangle $\Delta C_{2} A B$. The known distances $D_{1}$ and $D_{3}$, and angle $\theta_{1}$ are utilized to calculate $D$ as expressed by Eq. (9).

$$
D=\sqrt{\left(D_{1}^{2}+D_{3}^{2}-2 D_{1} D_{3} \cos \theta_{1}\right)}
$$

The knowledge of distance $D$ and last known location $\left(x_{i}^{\prime}, y_{i}^{\prime}\right)$ can be utilized to derive the equation of the circle $C_{2}$ as expressed by Eq. (10).

$$
\left(x-x_{i}^{\prime}\right)^{2}+\left(y-y_{i}^{\prime}\right)^{2}=D^{2}
$$

Similarly, the equation of line $l_{3}$ which passes through the last know location $\left(x_{i}^{\prime}, y_{i}^{\prime}\right)$ with direction $d_{i}^{\prime}$ can be derived as expressed by Eq. (11).

$$
\left(y-y_{i}^{\prime}\right)=d_{i}^{\prime}\left(x-x_{i}^{\prime}\right)
$$

By using Eq. (11), Eq. (10) can be simplified into two forms of quadratic equations as expressed by Eq. (12) and (13).

$$
\begin{aligned}
& x^{2}-2 x x_{i}^{\prime}+\left(x_{i}^{\prime}\right)^{2}-\frac{D^{2}}{1+\left(d_{i}^{\prime}\right)^{2}}=0 \\
& \text { and, } \quad y^{2}-2 y y_{i}^{\prime}+\left(y_{i}^{\prime}\right)^{2}-\frac{D^{2}\left(d_{i}^{\prime}\right)^{2}}{1+\left(d_{i}^{\prime}\right)^{2}}=0
\end{aligned}
$$

The two quadratic equations are solved to obtain the values of $(x, y)$ as expressed by Eq. (14).

$$
(x, y)=\left(x_{i}^{\prime} \pm \frac{D}{\sqrt{1+\left(d_{i}^{\prime}\right)^{2}}}, y_{i}^{\prime} \pm \frac{D \times d_{i}^{\prime}}{\sqrt{1+\left(d_{i}^{\prime}\right)^{2}}}\right)
$$

Further, the current location $\left(x_{i}, y_{i}\right)$ is a point on circle $C_{2}$. Therefore, the location satisfies Eq. (10) of circle $C_{2}$ as expressed by Eq. (15).

$$
\left(x_{i}-x_{i}^{\prime}\right)^{2}+\left(y_{i}-y_{i}^{\prime}\right)^{2}=D^{2}
$$

Similarly, the equation of line $l_{2}$ which passes through the current location $\left(x_{i}, y_{i}\right)$ with direction $d_{i}$ can be derived as expressed by Eq. (16).

$$
\left(y-y_{i}\right)=d_{i}\left(x-x_{i}\right)
$$

The equation of line $l_{2}$ is simplified using Eq. (14). The simplified equation of line $l_{2}$ can be expressed as given by Eq. (17).

$$
\begin{gathered}
\left(y_{i}^{\prime} \pm \frac{D \times d_{i}^{\prime}}{\sqrt{\left(1+d_{i}^{\prime 2}\right)}}-y_{i}\right)=d_{i}\left(x_{i}^{\prime} \pm \frac{D}{\sqrt{\left(1+d_{i}^{\prime 2}\right)}}-x_{i}\right) \\
\left(y_{i}^{\prime}-y_{i}\right)=d_{i}\left(x_{i}^{\prime}-x_{i}\right) \pm \frac{D \times d_{i}}{\sqrt{\left(1+d_{i}^{\prime 2}\right)}} \mp \frac{D \times d_{i}^{\prime}}{\sqrt{\left(1+d_{i}^{\prime 2}\right)}} \\
=d_{i}\left(x_{i}^{\prime}-x_{i}\right) \pm K
\end{gathered}
$$

where $K=\frac{D \times d_{i}}{\sqrt{\left(1+d_{i}^{\prime 2}\right)}} \mp \frac{D \times d_{i}^{\prime}}{\sqrt{\left(1+d_{i}^{\prime 2}\right)}}$ represents a constant value. The equation (15) of circle $C_{2}$ is simplified using Eq. (17) to obtain a quadratic equation as expressed by Eq. (18).

$$
\begin{aligned}
& \left(x_{i}-x_{i}^{\prime}\right)^{2}+\left\{d_{i}\left(x_{i}^{\prime}-x_{i}\right) \pm K\right\}^{2}-D^{2}=0 \\
& \left(x_{i}-x_{i}^{\prime}\right)^{2}\left(1+d_{i}^{2}\right) \mp 2 K d_{i}\left(x_{i}^{\prime}-x_{i}\right) \pm K^{2}-D^{2}=0
\end{aligned}
$$


$x_{i}^{2}+x_{i}\left(-2 x_{i}^{\prime} \pm \frac{2 K d_{i}}{\left(1+d_{i}^{2}\right)}\right)+\left(x_{i}^{\prime}\right)^{2} \mp \frac{2 K d_{i} x_{i}^{\prime}}{\left(1+d_{i}^{2}\right)} \pm \frac{K^{2}}{\left(1+d_{i}^{2}\right)}-\frac{D^{2}}{\left(1+d_{i}^{2}\right)}=0(18)$

Similarly, another quadratic equation is obtained from Eq. (15) of circle $C_{2}$ using Eq. (17). The quadratic equation can be expressed as given by Eq. (19).

$\left(y_{i}-y_{i}^{\prime}\right)^{2}+\left\{\frac{\left(y_{i}^{\prime}-y_{i}\right) \mp K}{d_{i}}\right\}^{2}-D^{2}=0$

$\left(y_{i}-y_{i}^{\prime}\right)^{2}\left(1+d_{i}^{2}\right) \mp 2 K\left(y_{i}^{\prime}-y_{i}\right) \pm K^{2}-d_{i}^{2} D^{2}=0$

$\left(y_{i}-y_{i}^{\prime}\right)^{2} \mp \frac{2 K\left(y_{i}^{\prime}-y_{i}\right)}{\left(1+d_{i}^{2}\right)} \pm \frac{K^{2}}{\left(1+d_{i}^{2}\right)}-\frac{d_{i}^{2} D^{2}}{\left(1+d_{i}^{2}\right)}=0$

$y_{i}^{2}+y_{i}\left(-2 y_{i}^{\prime} \pm \frac{2 K}{\left(1+d_{i}^{2}\right)}\right)+\left(y_{i}^{\prime}\right)^{2} \mp \frac{2 K y_{i}^{\prime}}{\left(1+d_{i}^{2}\right)} \pm \frac{K^{2}}{\left(1+d_{i}^{2}\right)}-\frac{d_{i}^{2} D^{2}}{\left(1+d_{i}^{2}\right)}=0$

The quadratic Eq. (18) and (19) are solved to obtain the current location of the vehicle $v_{i}$ as expressed by Eq. (20).

$$
\begin{array}{r}
\left(x_{i}, y_{i}\right)=\left(x_{i}^{\prime} \pm \sqrt{1 \pm\left(1+d_{i}^{2}\right) K^{2}-\left(1+d_{i}^{2}\right) D^{2}}, y_{i}^{\prime} \pm\right. \\
\left.\sqrt{1 \pm\left(1+d_{i}^{2}\right) K^{2}-\left(1+d_{i}^{2}\right) D^{2}}\right)(20)
\end{array}
$$

\section{Case-III: Location Estimation on Angular Road Sections}

In the third case, $d_{i} \perp d_{i}^{\prime}$ or $d_{i} \cong d_{i}^{\prime}$ after passing some perpendicular road sections, a vehicle $v_{i}$ receives GPS signal while moving on a road section. After a period, the vehicle passes through multiple angular road sections of perpendicular direction. It experiences GPS outage problem on a section as depicted in Fig. 6. In this situation, the problem of estimation of present location while passing on more than two angular road sections, is converted into a geometrical problem as depicted in Fig. 7. In generalized case of this geometrical problem, i.e., $N_{r s}$ number of road sections, the past heading distance $D_{H}^{\prime}$, and present heading distance $D_{H}$ can be calculated as given by Eq. (21) and (22).

$$
D_{H}^{\prime}=\sum_{i=1}^{\left\lceil\frac{N_{r s}}{2}\right]} D_{2 i-1}, \forall i \in\left\{1,2,3, \ldots\left\lceil\frac{N_{r s}}{2}\right\rceil\right\}
$$

and,

$$
D_{H}=\sum_{i=1}^{\left[\frac{N_{r s}}{2}\right]} D_{2 i}, \forall i \in\left\{1,2,3, \ldots\left\lfloor\frac{N_{r s}}{2}\right]\right\}
$$

Where the travel distance on each road section can be estimated as $D_{i}=\frac{\left(t_{i}-t_{i}^{\prime}\right)}{N_{r s}} \times \frac{\left(s_{i}+s_{i}^{\prime}\right)}{2}$ considering average speed and travel time. By utilizing the past and present heading distance, distance $D$ between past $\left(x_{i}^{\prime}, y_{i}^{\prime}\right)$ and present $\left(x_{i}, y_{i}\right)$ locations can be expressed as given by Eq. (23).

$$
D=\sqrt{{D_{H}^{\prime}}^{2}+D_{H}^{2}-2 D_{H}^{\prime} D_{H}}, \quad \theta_{3}=90^{\circ}
$$

The generalized geometrical problem can be solved, by considering a circle $C_{4}$ with radious of length $D$ and two lines $l_{4}$ and $l_{5}$ towards the past and present heading directions, respectively. The solution steps are quite similar to the steps followed in case-II for estimating present location derived in Eq. (20). The consideration of $+/$ - for location coordinate depends on the association of current direction of the vehicle with four poles of polar coordinate system.

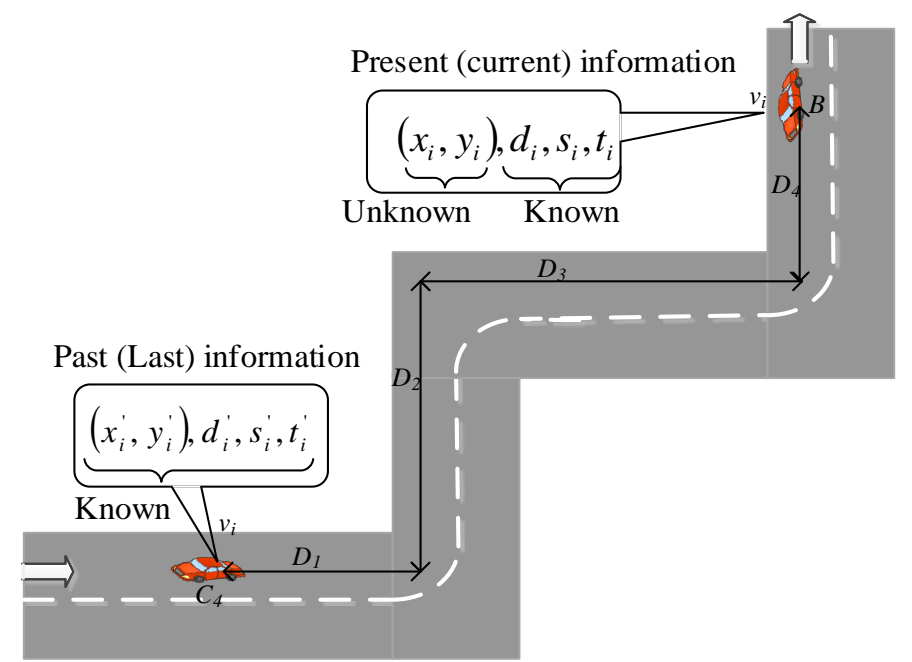

Fig. 6. GPS outage problem on multiple road sections

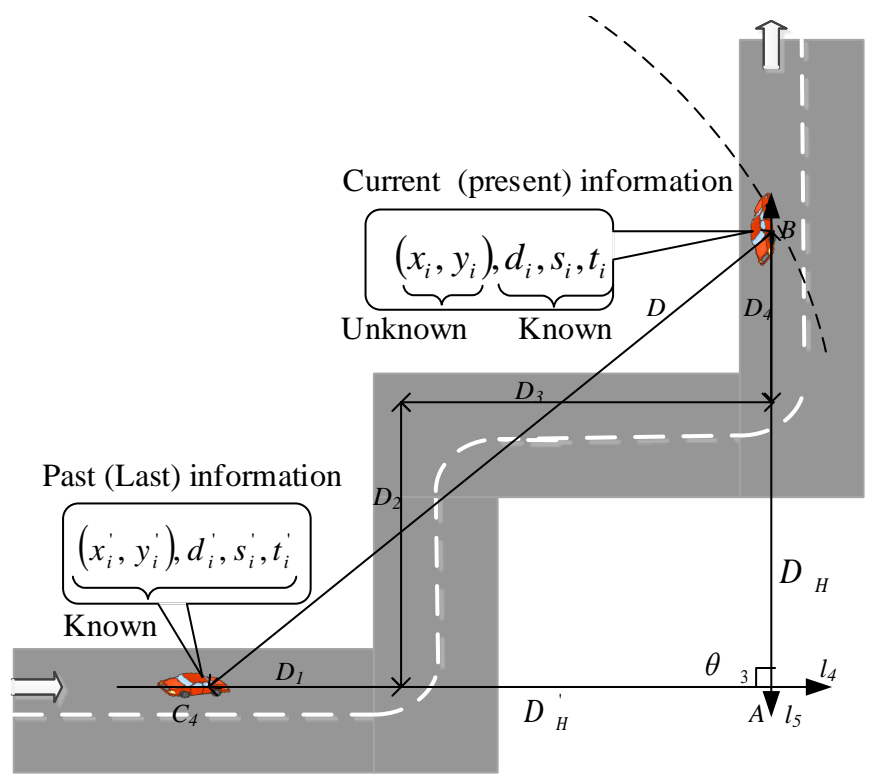

Fig. 7. Geometrical problem on multiple road sections

\section{Relocation}

The static and dynamic relocations are proposed to reduce the impact of GPS outage on location-based services.

\section{1) Static Relocation}

The vehicles belonging to the geographical area of the transmission range of source and destination pair, perform static relocation for a location-based service affected from GPS outage. The determination of current location of source and destination pair of a location-based service is the primary goal of static relocation. It is related to the specific geographical region. The vehicles going out of the region leave the duty, and the vehicles entering the region join the duty of static relocation. It is activated by the source and destination pair of a location-based service affected from GPS outage by sending land mark request. The neighboring vehicles accept the request, and send land mark reply. The request includes the geographical region, past and present direction, speed, and expected lifetime $E_{l t}$ of static relocation. The expected lifetime is calculated using relative information as expressed by Eq. (24). 


$$
E_{l t}=\frac{D_{r}}{\frac{s_{i}}{s_{\max }} \times \frac{s_{r}}{s_{\max }} \times \frac{d_{r}}{d_{\max }}}=\frac{\sqrt{\left(x_{s}-x_{d}\right)^{2}+\left(y_{s}-y_{d}\right)^{2}}}{\frac{s_{i}}{s_{\max }} \times \frac{\left|s_{s}-s_{d}\right|}{s_{\max }} \times \frac{\left|d_{s}-d_{d}\right|}{d_{\max }}}
$$

Where $D_{r}, s_{r}, d_{r}$ represent the distance, relative speed and direction of source and destination pair of a location-based service. The speed and direction limit is $s_{\max }$ and $d_{\max }$, respectively. The location of source and destination is represented by $\left(x_{s}, y_{s}\right)$ and $\left(x_{d}, y_{d}\right)$, respectively. Similarly, $s_{s}$ and $s_{d}$ represent the speeds, and $d_{s}$ and $d_{d}$ denote the direction of source and destination pair. The static relocation is wiped out either by a recipient using $E_{l t}$ or by the source and destination pair of the location-based service.

\section{2) Dynamic Relocation}

In a communication path of a location-based service affected from GPS outage, the vehicles within the transmission range of an intermediate vehicle are considered for dynamic relocation. It is performed by a designated intermediate vehicle, which is selected from neighbors for a location-based service. The selection is based on vehicular dynamics of each neighboring vehicle. It includes current direction $d_{i}$, speed $s_{i}$, number of directional change $N_{d_{i}}$ and number of speed change $N_{s_{i}}$ in the past. The intermediate vehicle sends dynamic relocation request to its neighbor. Each neighboring vehicle replies the request with their vehicular dynamics.

A cumulative weight for dynamic relocation $\mathbb{W}_{D R}$ is calculated for each neighbor, and the one having the maximum $\mathbb{W}_{D R}$ is selected for dynamic relocation. The four weight components of vehicular dynamics include weight of direction $\mathcal{W}_{d_{i}}$, speed $\mathcal{W}_{s_{i}}$, number of direction change $\mathcal{W}_{N_{d_{i}}}$, and number of speed change $\mathcal{W}_{N_{s_{i}}}$. Each component of the vehicular dynamics is assigned with an individual weight to calculate $\mathbb{W}_{D R}$. The weight of direction is calculated considering the relative difference in direction between the intermediate and neighbor vehicles as expressed by Eq. (25).

$$
\mathcal{W}_{d_{i}}= \begin{cases}1, & \left|d_{\text {in }}-d_{i}\right| \cong 0^{\circ} \\ 0, & \left|d_{i n}-d_{i}\right| \cong 180^{\circ} \\ \alpha^{\left|d_{i n}-d_{i}\right|}, & \left|d_{i n}-d_{i}\right| \leq 180^{\circ} \\ 1-\alpha^{\left|d_{i n}-d_{i}\right|}, & \left|d_{i n}-d_{i}\right|>180^{\circ}\end{cases}
$$

where $d_{i n}$ and $d_{i}$ represent the direction of the intermediate and $i^{\text {th }}$ neighbor, respectively, and $\alpha$ represents the decisive factor of direction. The first two cases, $\left|d_{i n}-d_{i}\right| \cong 0^{\circ}$ and $\left|d_{\text {in }}-d_{i}\right| \cong 180^{\circ}$ represent equal and opposite direction, respectively. The third case, $\left|d_{i n}-d_{i}\right| \leq 180^{\circ}$ represents the constraint $\left(0 \leq d_{i n}, d_{i} \leq 180\right)$ or $\left(180 \leq d_{i n}, d_{i} \leq 360\right)$. The fourth case represents the constraint $\left(0 \leq d_{\text {in }}\right.$ or $d_{i} \leq$ $180)$ and $\left(180 \leq d_{\text {in }}\right.$ or $\left.d_{i} \leq 360\right)$. An illustrative example is presented in Fig. 6. Similarly, the weight of speed $\mathcal{W}_{s_{i}}$ is calculated based on the ratio of speed between neighboring and the intermediate vehicles, as expressed by Eq. (26).

$$
\mathcal{W}_{s_{i}}=\left\{\begin{array}{lr}
1, & \frac{s_{i}}{s_{\text {in }}} \cong 1 \\
0, & \frac{s_{i}}{s_{\text {in }}} \geq 2 \\
\beta^{\frac{s_{i}}{s_{\text {in }}},} & 1<\frac{s_{i}}{s_{\text {in }}}<2
\end{array}\right.
$$

where $s_{\text {in }}$ and $s_{i}$ represent the speed of intermediate and $i^{\text {th }}$ neighbor, respectively, and $\beta$ represents the decisive factor of speed. The weight of direction change and speed change, are calculated as expressed by Eq. (27) and (28), respectively.

$$
\begin{aligned}
\mathcal{W}_{N_{d_{i}}} & =1-\gamma^{N_{d_{i}}} \\
\text { and, } \quad \mathcal{W}_{N_{s_{i}}} & =1-\delta^{N_{s_{i}}}
\end{aligned}
$$

where $\gamma$ represents the decisive factor of $N_{d_{i}}$, and $\delta$ represents the decisive factor of $N_{s_{i}}$. The four decisive factors $\alpha, \beta, \gamma$ and $\delta$ are given successive decreasing importance considering the relative impact of the parameters on vehicular dynamics. The cumulative weight $\mathbb{W}_{D R}$ is calculated for each neighboring vehicle by adding these weight components of vehicular dynamics as expressed by Eq. (29).

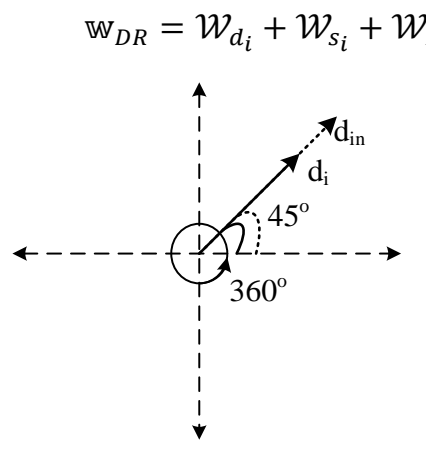

(a)

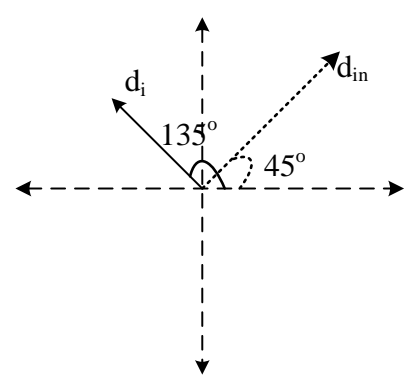

(c)

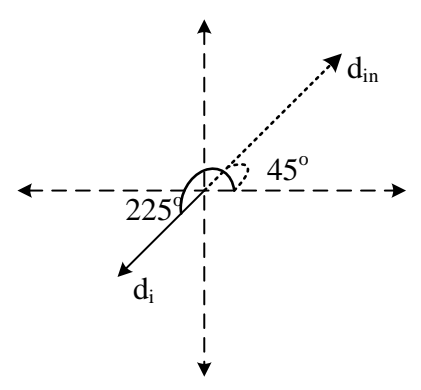

(b)

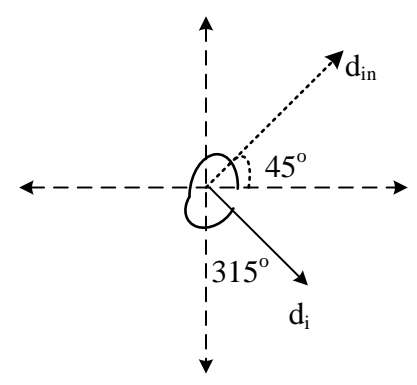

(d)
Fig. 6. (a) $\mathcal{W}_{d_{i}}=1,\left|45^{\circ}-45^{\circ}\right|=0^{\circ}$, (b) $\mathcal{W}_{d_{i}}=0,\left|225^{\circ}-45^{\circ}\right|=180^{\circ}$, (c) $\mathcal{W}_{d_{i}}=\alpha^{90},\left|135^{\circ}-45^{\circ}\right|=90^{\circ}$, (d) $\mathcal{W}_{d_{i}}=1-\alpha^{270},\left|315^{\circ}-45^{\circ}\right|=$ $270^{\circ}$

\section{EMPIRICAL RESULTS}

In this section, analytical and case study based simulation results are discussed for analyzing the performance of the proposed geometry based vehicular localization system. This section is broadly divided into three parts. In the first part, analytical results are discussed. In the second part, simulation setting and comparative analysis of results are discussed. A summary of observations is presented in the third part.

\section{A. Analytical Results}

The characteristics of the mathematical derivations are analytically analyzed using mathematical tool. The analytical analysis evaluates the performance of mathematical formulations obtained for addressing GPS outage problem in VCPS environments. Most of the parameters considered for generating analytical results are mentioned with result itself. 
The major parameters include the distance $D$ travelled during GPS outage time, current direction $d_{i}$, and expected lifetime of relocation $E_{l t}$.
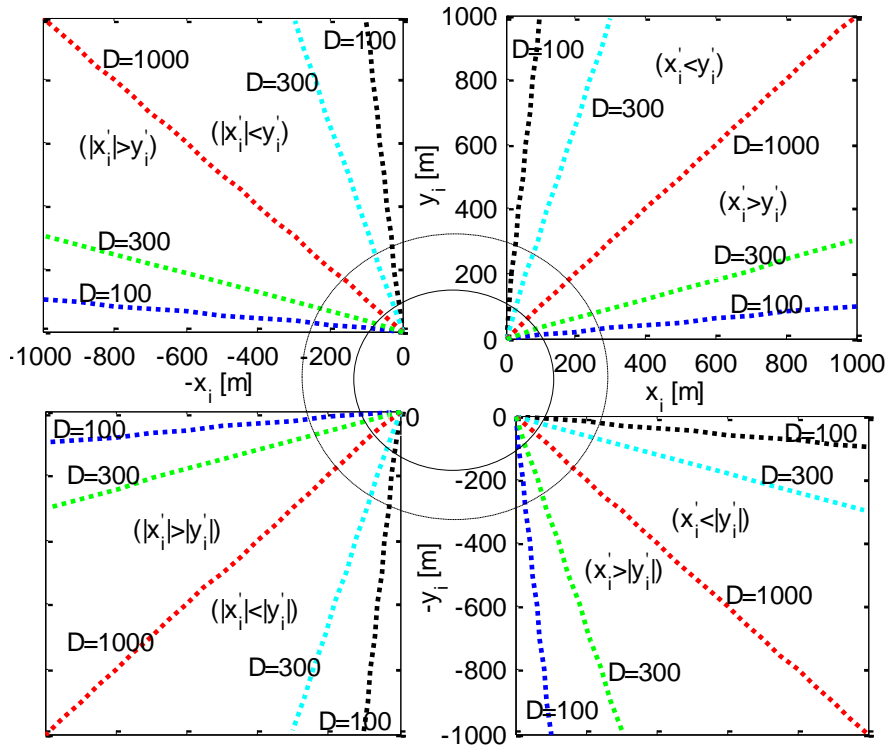

Fig. 7. Impact of $D$ on location estimation, shorter GPS outage of case I

The analytical evaluation of the location estimation for shorter GPS outage under case I, i.e., $d_{i} \cong d_{i}^{\prime}$ or $\left|d_{i}-d_{i}^{\prime}\right| \cong$ $180^{\circ}$ is presented in Fig.7. The two directions $0^{\circ}$ and $180^{\circ}$ are considered for generating results under varying distance $D$ in range [100 1000]. The impact of $D$ on the current location $\left(x_{i}, y_{i}\right)$ is analyzed considering locations on a polar coordinate system, i.e., $\left( \pm x_{i}, \pm y_{i}\right)$. The following two observations can be made from results. Firstly, the current location follows linear order displacement with respect to the last known location. The linear order displacement is clearly verifiable from results, as the current location $\left(x_{i}, y_{i}\right)$ follows straight lines in each pole of the polar coordinate system. Secondly, the impact of $D$ on the current location $\left(x_{i}, y_{i}\right)$ is sharper with larger distance $D$, i.e., [300, 1000]. This is evident in results as the angle of displacement or the angle of straight lines with respect to axis increases with the increment in $D$. It is also noteworthy that impact of $D$ on the current location $\left(x_{i}, y_{i}\right)$ is unilaterally equal in each pole of the coordinate system. The two circles centered at the last known position $\left(\mathrm{x}_{i}^{\prime}, y_{i}^{\prime}\right)$ cut at equal distance on the straight lines (representing the estimated current positions with distance $D$ ), as the radius of the circles in each pole of the coordinate system.

The analytical evaluation of the location estimation for longer GPS outage under case II, i.e., $d_{i} \neq d_{i}^{\prime}$ and $\left|d_{i}-d_{i}^{\prime}\right| \neq$ $180^{\circ}$, is presented in Fig.8. The evaluation focuses on the impact of difference in direction $\left|d_{i}-d_{i}^{\prime}\right|$ on the estimation of current location $\left(x_{i}, y_{i}\right)$. In this case also, the estimation of current $\left(x_{i}, y_{i}\right)$ is analyzed in a polar coordinate system considering locations at the four poles. The positive and negative sides of both $\mathrm{x}$-axis and $\mathrm{y}$-axis are considered, which are represented by $x_{i}$ and $y_{i}$, respectively. The z-axis represents the difference in direction $\left|d_{i}-d_{i}^{\prime}\right|$ in terms of degree. The following two observations are made from results.
Firstly, the impact of $\left|d_{i}-d_{i}^{\prime}\right|$ on the current location $\left(x_{i}, y_{i}\right)$ increases with the increase in $\left|d_{i}-d_{i}^{\prime}\right|$ up to $90^{\circ}$. The impact of $\left|d_{i}-d_{i}^{\prime}\right|$ on current location $\left(x_{i}, y_{i}\right)$ reduces with the further increase in $\left|d_{i}-d_{i}^{\prime}\right|$ towards $180^{\circ}$. This is due the conversion of location estimation from case II to case I with $\left|d_{i}-d_{i}^{\prime}\right|$ approaching towards $180^{\circ}$. Secondly, it is also notable that the impact of $\left|d_{i}-d_{i}^{\prime}\right|$ on the current location $\left(x_{i}, y_{i}\right)$ is unilaterally equal in all the four poles of the coordinate system. This can be attributed to the fact that the four corners of the two rectangles point the surfaces (represents the estimated current positions with specific direction), which are equal in height in all the four poles.

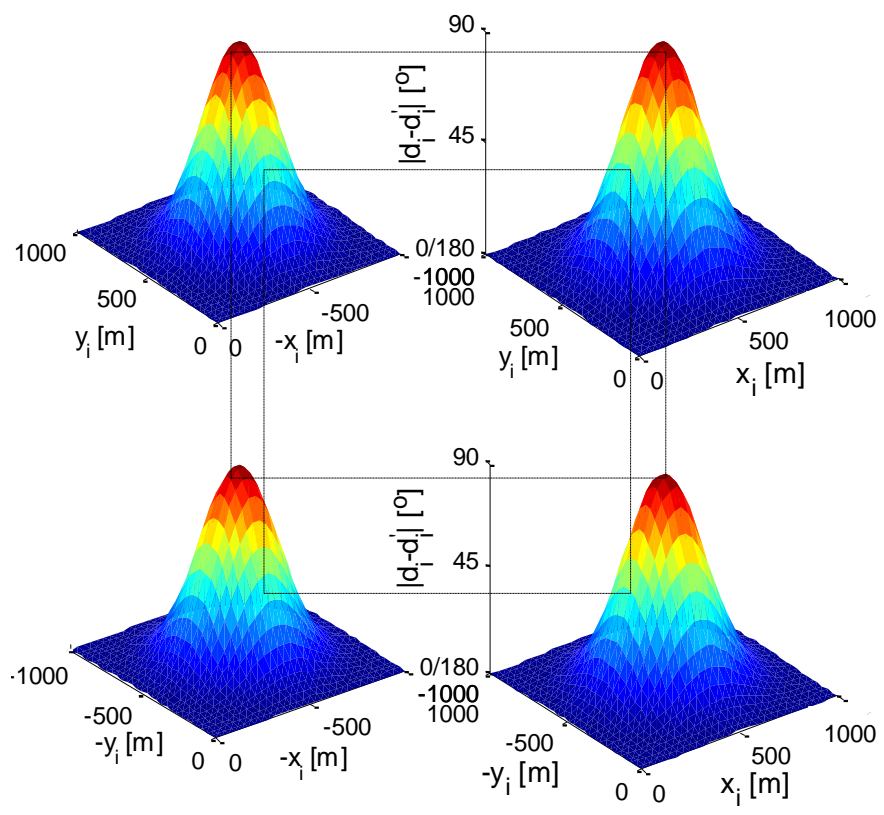

Fig. 8. Impact of $\left|d_{i}-d_{i}^{\prime}\right|$ on location estimation under case 2

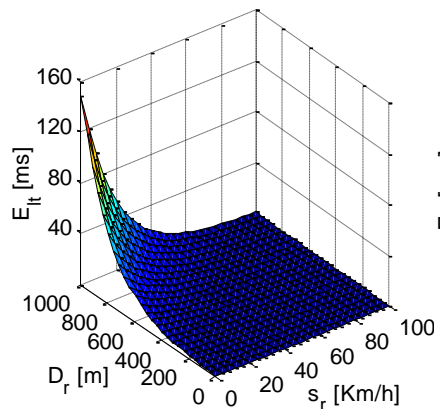

(a)

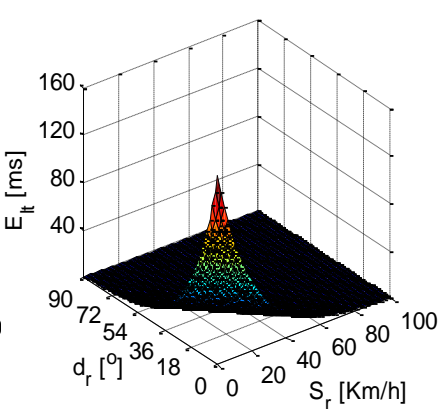

(b)
Fig. 9. Impact of parameters on $E_{l t}$, (a) $D_{r}$ and $s_{r}$, (b) $d_{r}$ and $s_{r}$

The analytical evaluation of static relocation is presented in Fig. 9. The impact of relative distance $\mathrm{D}_{r}$ and speed $s_{r}$ on expected lifetime $E_{l t}$ is shown in Fig. 9(a). The impact of relative direction $d_{r}$ and speed $s_{r}$ on expected lifetime $E_{l t}$ is shown in Fig. 9(b). Form the close observation of both results, the following two points are noteworthy. Firstly, the sharp increment in expected lifetime $E_{l t}$ with larger relative distance $D_{r}$ and lower relative speed $s_{r}$. Secondly, the sharp decrement in expected lifetime $E_{l t}$ with larger relative direction $d_{r}$ and higher relative speed $s_{r}$. The observations are clearly verifiable from the mathematical derivation of $E_{l t}$. The longer expected lifetime with the larger relative distance 
and lower relative speed reduces the impact of GPS outage time on the location-based services. The shorter expected lifetime with the larger relative direction and higher relative speed enhances the efficiency of location-based services during GPS outage.
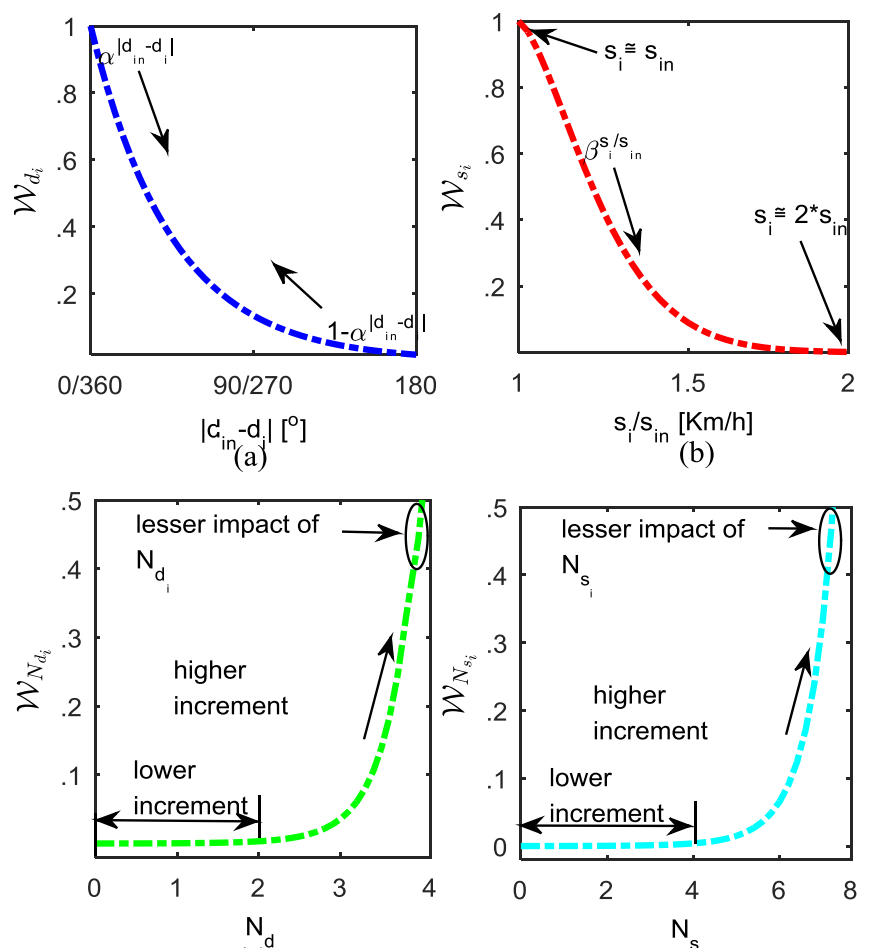

(c) d $^{i}$

$(\mathrm{d})^{\mathrm{i}}$

Fig. 10. Impact of parameter on weight component, (a) $\mathcal{W}_{d_{i}}$ versus $\left|d_{i n}-d_{i}\right|$, (b) $\mathcal{W}_{s_{i}}$ versus $\frac{s_{i}}{s_{i n}}$ (c) $\mathcal{W}_{N_{d_{i}}}$ versus $N_{d_{i}}$, (d) $\mathcal{W}_{N_{s_{i}}}$ versus $N_{s_{i}}$

The analytical evaluation of dynamic relocation is presented in Fig. 10. The results in Fig. 10(a) highlight the impact of $\left|d_{i n}-d_{i}\right|$ on the weight of direction $\mathcal{W}_{d_{i}}$. It can be clearly observed that the weight poses the highest value, when the intermediate, and neighbor vehicles have same driving direction, i.e., $\left|d_{i n}-d_{i}\right|=0^{\circ}$ or $360^{\circ}$. Further, the weight $\mathcal{W}_{d_{i}}$ decreases with the increase in difference in driving direction of both the vehicles. The decrement is represented by the third case of the derivation of $\mathcal{W}_{d_{i}}$, i.e., $\alpha^{\left|d_{i n}-d_{i}\right|}$. The reduction in the difference in driving direction results as the increment in the weight $\mathcal{W}_{d_{i}}$, which is represented by the fourth case of the derivation of $\mathcal{W}_{d_{i}}$, i.e., $1-\alpha^{\left|d_{i n}-d_{i}\right|}$. The weight poses the lowest value, when the intermediate and neighbor vehicles have exactly opposite driving direction, i.e., $\left|d_{i n}-d_{i}\right|=180^{\circ}$. The impact of ratio of speed on the weight of speed $\mathcal{W}_{s_{i}}$ is shown in Fig. 10(b). It can be clearly observed that the weight poses highest value, when the intermediate and neighbor vehicles have equivalent driving speed, i.e., $\frac{s_{i}}{s_{i n}}=1$. Moreover, the weight $\mathcal{W}_{s_{i}}$ decreases with the increase in ratio of speed between the vehicles. The decrement is represented by the third case of the derivation of $\mathcal{W}_{s_{i}}$, i.e., $\beta^{\frac{s_{i}}{s_{i n}}}$. The weight poses the lowest value, when the ratio of speed between neighbor and the intermediate vehicles reaches to two or more.
The impact of number of direction changes on the corresponding weight is presented in Fig. 10(c). It is noteworthy that the weight $\mathcal{W}_{N_{d_{i}}}$ increases marginally with two or fewer direction change. The increment is very sharp with more than two direction changes, particularly with three, four direction changes. It is also notable that the impact of $N_{d_{i}}$ on the weight reduces sharply when the weight reaches higher than 0.4. The impact of number of speed changes on the corresponding weight is presented in Fig. 10(d). It can be observed that the weight increases marginally with four or fewer speed changes. The increment is very sharp with more than four speed changes, particularly with six and eight speed changes. It is also noteworthy that the impact of $N_{d_{i}}$ on the weight reduces sharply when the weight reaches higher than 0.4 , which is quite similar to the case of number of direction changes.

\section{B. Case Study}

In this section, simulations carried out to evaluate the performance of GeoLV is presented focusing on environment setting, metrics, and comparative analysis of results. The following two objectives were aimed for performing case study based simulations. First, to evaluate the effectiveness and efficiency of GeoLV in handling GPS outage problem, in comparison with the state-of-the-art techniques including GOT [19] and IVCAL [22] considering real road network. Second, to measure the impact of GPS outage time and density of vehicles on the performance of GeoLV and the state-of-theart techniques.

\section{1) Simulation Setting and Metrics}

Simulations are carried out using network simulator ns-2 with supporting applications including mobility model generation for vehicular environment (MOVE) [24] and a geographic information system known as ArcGIS [25]. MOVE which is developed on the top of simulation for urban mobility, is utilized for generating realistic vehicular network scenario on the real road map of Kuala Lumpur, Malaysia (see. Fig.11). It is worth noting that the capital city is full of high rise buildings alongside roads, and thus, GPS outage is a serious issue in the city. The two main modules of MOVE namely, road map editor, and vehicle movement editor are utilized to create realistic vehicular scenario. The vehicular scenario features number of vehicles, speed of vehicles, speed range on a specific road section, flows in each lane, traffic lights at junctions, and probability of turning left or right for vehicles at junctions. ArcGIS is utilized to embed twodimensional location coordinates on the city road map, which is obtained via open street map web application.

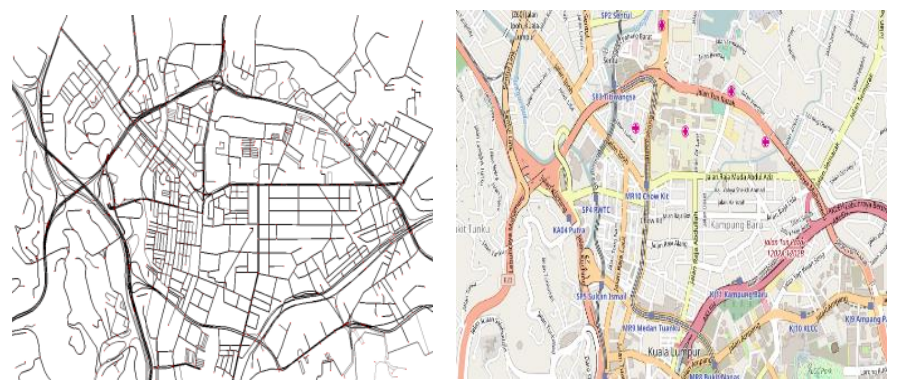

Fig. 11. Simulation scenario of Kuala Lumpur 
The three types of road structures are the focus of experiments. Firstly, the vehicles passing through the straight roads are considered to evaluate the localization capability of GeoLV under shorter GPS outage problem of case I. Secondly, the vehicles passing through curve and angular roads are considered to examine the localization capability under longer GPS outage of case II and III. Thirdly, in hybrid road environment, some vehicles are selected from straight roads and some from curved and angular roads to assess the impact of static and dynamic relocations on the performance of location-based services.

On the city road map, [200 1000] vehicles are considered moving with the speed range $[20 \sim 80 \mathrm{~km} / \mathrm{h}]$. For testing location estimation under the three cases, vehicles moving on city scenario periodically record their locations at the rate of $1 s$. For testing static and dynamic relocations, vehicles record their locations as well as communicate with the location-based service running on designated vehicles. For simulating GPS outage, the vehicles passing through the specific type of road sections including straight, curved, and angular with high rise building alongside roads, stop using ArcGIS embedded GPS locations, and start using GeoLV calculated locations for periodic recording. The random switch-off from ArcGIS embedded location recording is also considered for simulating random GPS outage problem, without considering the road sections. As signal propagation model for communication, shadowing is considered with path loss exponent 5 and shadowing deviation in the range [3.6 5.2db]. Following successive decreasing importance, the considered decisive factors include $\alpha=0.75, \beta=0.187, \gamma=0.046$, and $\delta=$ 0.017 with the constraint $(\alpha+\beta+\gamma+\delta=1)$. For other general parameter setting, readers are suggested to follow the parameter table considered in [26].

As localization performance metrics, location error is measured when vehicles pass through the point of interest road sections including straight, curved, or angular roads. It is the difference between ArcGIS embedded GPS location and the location calculated using GeoLV. The localization delay is measured as the average successive location calculation time, during GPS outage on a specific road section. The impact of GPS outage time, and density of vehicles are considered for measuring both metrics on the city road map.

2) Analysis of Results

Impact of GPS Outage Time:

A comparison of the impact of GPS outage time on location error between GeoLV and the state-of-the-art techniques is presented in Fig. 12(a)-(d) considering different speed of vehicles. It can be clearly observed that the location error of GeoLV is lower and stable, whereas the error increases with longer GPS outage time in case of the state-of-the-art techniques. The observation affirms the capability of GeoLV in handling GPS outage. This can be attributed to the accurate location estimation of GeoLV on single and multiple road sections using geometry, with the knowledge vehicular dynamics and road trajectory. The location error of the stateof-the-art techniques increases with longer GPS outage time, i.e., [60 300s]. This is due to the relative distance based localization among neighboring vehicles in GOT and IVCAL. The relative distances from neighbors are erroneous due to the unavailability of neighbor locations in case of GPS outage. Moreover, fuzzy geometry based localization in GOT is more effective, than the communication assisted localization in IVCAL. It is also worth noting that the location error of GeoLV as well as the state-of-the-art techniques increases with higher speed of vehicles, i.e., [20 80 km/h]. Although the location error increment in GeoLV is lower than those of the state-of-the-art techniques. This is due to the road geometry based distance calculation in GeoLV, whereas location based distance calculation in GOT and IVCAL.

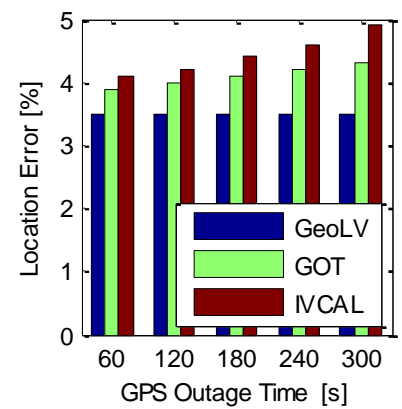

(a)

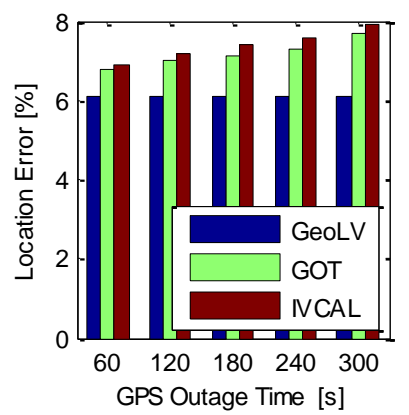

(c)

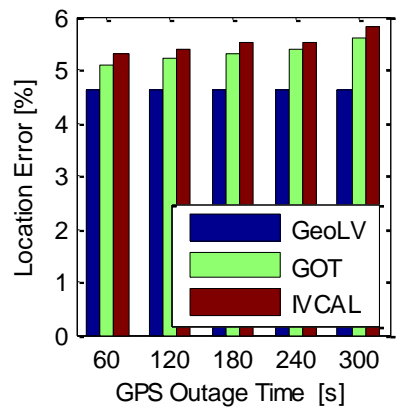

(b)

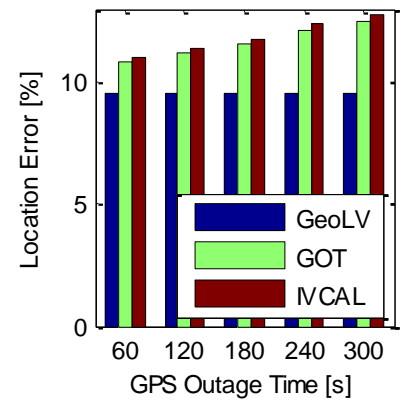

(d)
Fig. 12. Impact of GPS outage time on location error with (a) $v_{s}=20 \mathrm{~km} / \mathrm{h}$ (b) $v_{s}=40 \mathrm{~km} / \mathrm{h}$, (c) $v_{s}=60 \mathrm{~km} / \mathrm{h}$, (d) $v_{s}=80 \mathrm{~km} / \mathrm{h}$

A comparison of the impact of GPS outage time on localization delay between GeoLV and the state-of-the-art techniques is presented in Fig. 13(a)-(d) considering different speed of vehicles. It is evident that the localization delay of GeoLV is smaller and stable. It is not affected by the longer GPS outage time [60 300s] and higher speed of vehicles [20 80 km/h]. This is due to the geometry based localization using vehicular dynamics and road trajectory, without depending on neighboring vehicles for distance calculation. The localization delay of the state-of-the-art techniques is longer as compared to that of GeoLV. The reason is the dependency on neighboring vehicles for relative distance calculation. The communication delay among neighboring vehicles (for sharing location in case of GOT, and relative distance in case of IVCAL) increases localization delay of the state-of-the-art techniques. It is also notable that the localization delay of the state-of-the-art techniques becomes longer with higher speed of vehicles. This is due to the communication failure with higher speed of vehicles in case of GOT and IVCAL. 


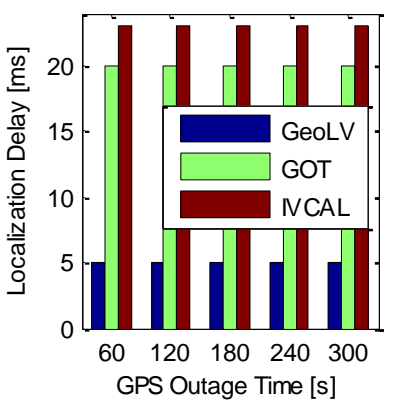

(a)

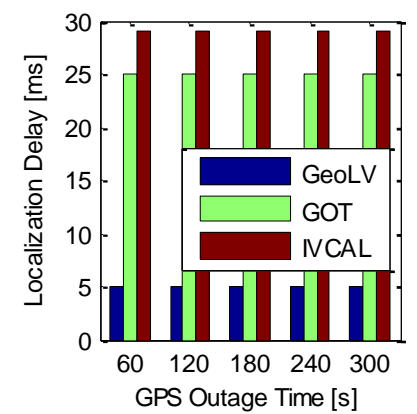

(c)

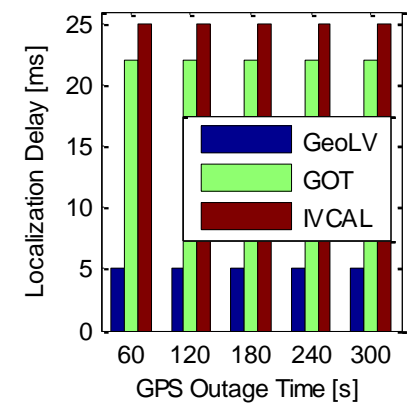

(b)

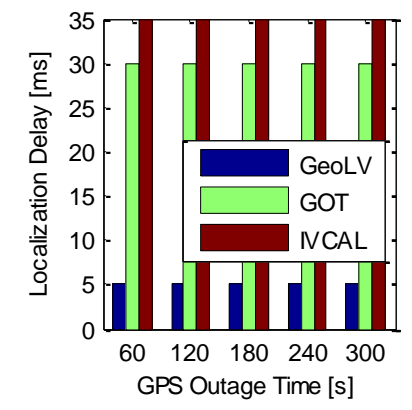

(d)
Fig. 13. Impact of GPS outage time on localization delay with (a) $v_{s}=$ $20 \mathrm{~km} / \mathrm{h}$ (b) $v_{s}=40 \mathrm{~km} / \mathrm{h}$, (c) $v_{s}=60 \mathrm{~km} / \mathrm{h}$, (d) $v_{s}=80 \mathrm{~km} / \mathrm{h}$

\section{Impact of Density of Vehicles:}

A comparison of the impact of density of vehicles on location error between GeoLV and the state-of-the-art techniques is presented in Fig. 14(a)-(d) considering different density of vehicles. It can be clearly observed that the location error of GeoLV is lower and stable. This can be attributed to the vehicular dynamics and road trajectory based selflocalization capability of GeoLV without using information from neighboring vehicles. The location error of the state-ofthe-art techniques decreases with higher density of vehicles, i.e., [200 1000]. This is due to the availability of location information in case of GOT, and relative distance information in case of IVCAL, from more neighboring vehicles. This improves location prediction, and thus, location error reduces. It is also worth noting that the location error of the state-ofthe-art techniques stabilize with 800 and higher vehicle density. This can be attributed to the satisfaction of availability of either location information and relative distance information, from maximum number of neighboring vehicles requirement. Thus, further increase in density has no impact on location error of the state-of-the-art techniques.

A comparison of the impact of density of vehicles on localization delay between GeoLV and the state-of-the-art techniques is presented in Fig. 15(a)-(d) considering different density of vehicles. It is evident from results that the localization delay of GeoLV is smaller and stable, due to the non-dependency on neighboring vehicles for localization. The localization delay of the state-of-the-art techniques increases with the increment in density of vehicles, i.e., [200 1000]. This is due to the involvement of higher number of neighboring vehicles in localization process. It ultimately causes delay in localization due to the communication delay among neighboring vehicles. It is also noteworthy that the minimum localization delay is constrained for considered speed of vehicle [20 80km/h], due to the error in calculating relative distance and location of neighboring vehicles.

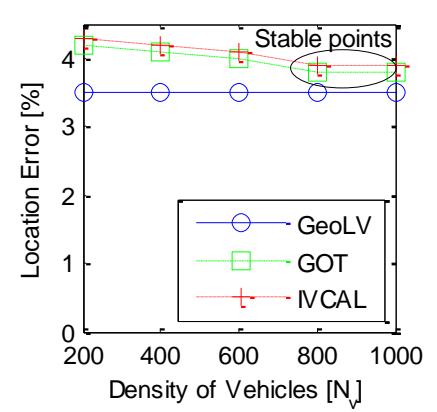

(a)

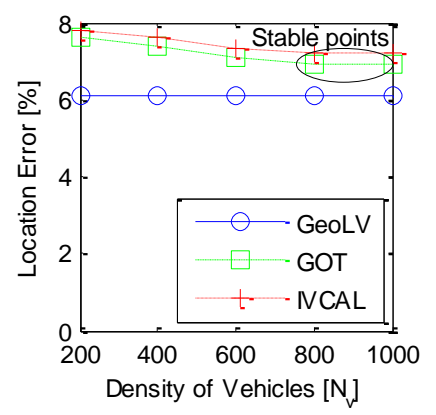

(c)

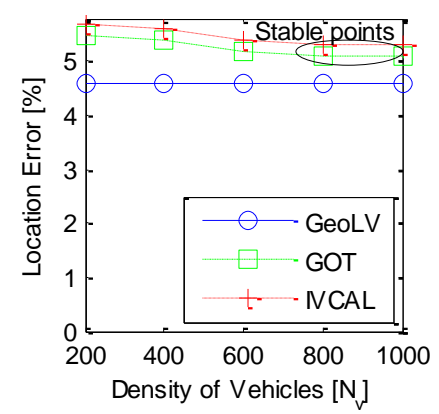

(b)

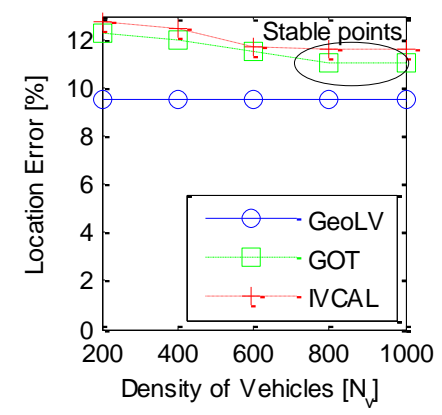

(d)
Fig. 14. Impact of density of vehicles on location error with (a) $v_{s}=20 \mathrm{~km} / \mathrm{h}$ (b) $v_{s}=40 \mathrm{~km} / \mathrm{h}$, (c) $v_{s}=60 \mathrm{~km} / \mathrm{h}$, (d) $v_{s}=80 \mathrm{~km} / \mathrm{h}$

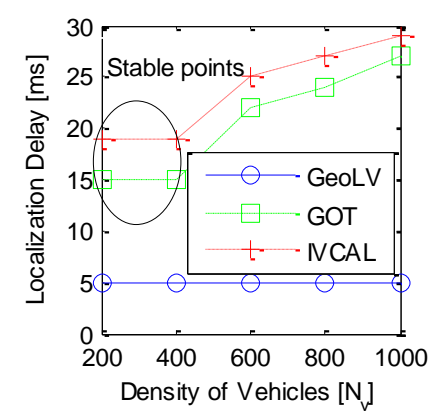

(a)

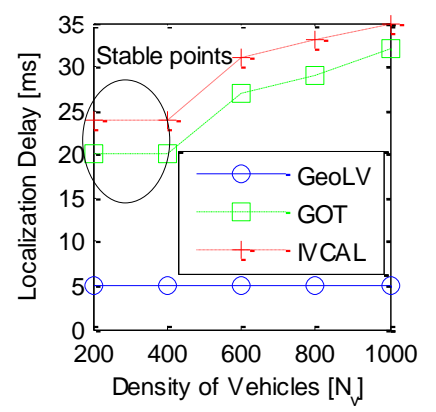

(c)

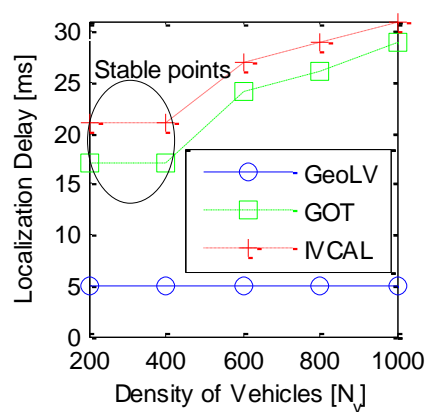

(b)

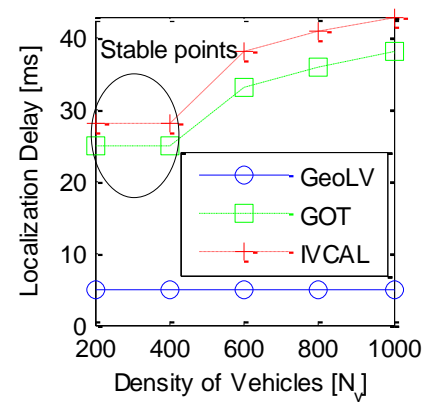

(d)
Fig. 15. Impact of density of vehicles on localization delay with (a) $v_{s}=$ $20 \mathrm{~km} / \mathrm{h}$ (b) $v_{s}=40 \mathrm{~km} / \mathrm{h}$, (c) $v_{s}=60 \mathrm{~km} / \mathrm{h}$, (d) $v_{s}=80 \mathrm{~km} / \mathrm{h}$

\section{Summary of Observations}

The following observations are made from the design, implementation and analysis of experimental results. The shorter GPS outage problem can be converted into geometrical problem on a straight road section. The problem on straight 
road section can be solved using vehicular dynamics. The longer GPS outage problem can be converted into geometrical problem on curved and angular road trajectories. The problem can be addressed using road trajectory along with vehicular dynamics. The harsh impact of GPS outage problem on location-based services can be effectively reduced using efficient static and dynamic relocations.

The analytical analysis of the proposed solution for shorter GPS outage problem affirms two points. Firstly, linear order displacement of current location with respect to the last known GPS location. Secondly, the sharper impact of larger travelling distance during GPS outage on current location. The analysis of the solution for longer GPS outage problem attests the higher impact of the increasing directional difference up to $90^{\circ}$, and lower impact with larger directional difference up to $180^{\circ}$. In static relocation, expected lifetime of relocation increases sharply with larger distance and lower relative speed. In dynamic relocation, weight component of direction poses the highest value with equal or opposite direction, and decreases with the increase in directional difference. The weight component of speed poses the highest value with equal speed, and decreases with the increase in ratio of speed.

As outage threshold, GeoLV predicts accurate location for the outage duration, till the vehicle travels on the same straight road in case I, or till the vehicle remains on curved road section in case II, or till the driving direction of the vehicle remains perpendicular or equal to the initial direction after passing adjoining angular road sections. In terms of procedural complexity, the execution of location prediction in road trajectory and past location based GeoLV is efficient than the neighboring vehicles location based prediction in GOT and IVCAL, due to the execution delay in communication with neighboring vehicles for location information in case of GOT and distance information in case of IVCAL.

\section{CONCLUSION AND FUTURE WORK}

In this paper, a geometry-based location estimation technique is presented for GPS outage problem in VCPS environments. The solution of GPS outage problem focuses on shorter and longer outage with different types of road trajectories including straight, curved and angular road sections. The shorter GPS outage problem on straight road section is solved using the knowledge of vehicular dynamics. The longer GPS outage problem on curved and angular road sections is solved utilizing the knowledge of road trajectories along with vehicular dynamics. The proposed geometry-based localization technique is useful for aiding various locationbased services in vehicular cyber physical systems environments. In future research, authors will be focusing on lane-level vehicular localization using geometrical concepts on tiles-based road network.

\section{REFERENCES}

[1] J. Wan, D. Zhang, S. Zhao, L.T. Yang, and J. Lloret, "Context-aware vehicular cyber-physical systems with cloud support: architecture, challenges, and solutions," IEEE Communications Magazine, vol. 52, no. 8, pp.106-113, 2014.

[2] O. Kaiwartya, A.H. Abdullah, Y. Cao, A. Altameem, M. Prasad, C.T. Lin and X. Liu, "Internet of Vehicles: Motivation, Layered Architecture, Network Model, Challenges and Future Aspects," IEEE Access, vol. 4, no.1, pp.5356-5373, 2016.
[3] Perallos, A., "Intelligent Transport Systems: Technologies and Applications," John Wiley \& Sons, Chichester, 2015.

[4] Papadimitratos, P., De La Fortelle, A., Evenssen, K., Brignolo, R. and Cosenza, S., "Vehicular communication systems: Enabling technologies, applications, and future outlook on intelligent transportation," IEEE Communications Magazine, 47(11), pp.84-95, 2009.

[5] Al-Sultan, S., Al-Doori, M.M., Al-Bayatti, A.H. and Zedan, H., "A comprehensive survey on vehicular Ad Hoc network," Journal of network and computer applications, 37, pp.380-392, 2014.

[6] Department of Transportation, US. "ITS Strategic Plan 2015-2019". Available: http://www.its.dot.gov/research_areas/strategicplan2015.htm

[7] Fawcett, J. and Robinson, P., "Adaptive routing for road traffic," IEEE Computer Graphics and Applications, 20(3), pp.46-53, 2000.

[8] Ammoun, S., Nashashibi, F. and Laurgeau, C., "Crossroads risk assessment using GPS and inter-vehicle communications," IET Intelligent Transport Systems, 1(2), pp.95-101, 2007.

[9] Wu, D., Zhang, Y., Bao, L., Regan, A.C., "Location-based crowdsourcing for vehicular communication in hybrid networks," IEEE transactions on intelligent transportation systems, 14(2),pp.837-846, 2013.

[10]Cui, Y. and Ge, S.S., "Autonomous vehicle positioning with GPS in urban canyon environments," IEEE transactions on robotics and automation,19(1), pp.15-25, 2003.

[11]Parker, R. and Valaee, S. "Vehicular node localization using receivedsignal-strength indicator," IEEE Transactions on Vehicular Technology, 56(6), pp.3371-3380, 2007.

[12] Cheng, W., Cheng, X., Song, M., Chen, B. and Zhao, W.W. "On the design and deployment of RFID assisted navigation systems for VANETs," IEEE Transactions on Parallel and Distributed Systems, 23(7), pp.1267-1274, 2012.

[13] Wang, J., Ni, D. and Li, K. "RFID-based vehicle positioning and its applications in connected vehicles," Sensors, 14(3), pp.4225-4238, 2014.

[14] Ou, C.H. "A roadside unit-based localization scheme for vehicular ad hoc networks," International Journal of Communication Systems, 27(1), pp.135-150, 2014.

[15] Khattab, A., Fahmy, Y., Wahab, A.A. "High accuracy GPS-Free vehicle localization framework via an INS-Assisted single RSU," International Journal of Distributed Sensor Networks, 15(1), pp.71-86, 2015.

[16]Boukerche, A., Oliveira, H.A., Nakamura, E.F. and Loureiro, A.A., "Vehicular ad hoc networks: A new challenge for localization-based systems," Computer communications, 31(12), pp.2838-2849, 2008.

[17]Zhou, J. and Shi, J., "A comprehensive multi-factor analysis on RFID localization capability," Advanced Engineering Informatics, 25(1), pp.3240, 2011.

[18] Vu, T.D., Burlet, J. and Aycard, O. "Grid-based localization and local mapping with moving object detection and tracking," Information Fusion,12(1), pp.58-69, 2011.

[19] Yan, T., Zhang, W. and Wang, G. "A grid-based on-road localization system in vanet with linear error propagation," IEEE Transactions on Wireless Communications, 13(2), pp.861-875, 2014.

[20]Alam, N., Balaei, A.T. and Dempster, A.G. "A DSRC Doppler-based cooperative positioning enhancement for vehicular networks with GPS availability," IEEE Transactions on Vehicular Technology, 60(9), pp.4462-4470, 2011.

[21] Alam, N., Balaei, A., Dempster, A.G. "Relative positioning enhancement in VANETs: A tight integration approach," IEEE Transactions on Intelligent Transportation Systems, 14(1), pp.47-55, 2013.

[22]Drawil, N.M. and Basir, O. "Intervehicle-communication-assisted localization," IEEE Transactions on Intelligent Transportation Systems, 11(3), pp.678-691, 2010.

[23] Li, H. and Nashashibi, F. "Cooperative multi-vehicle localization using split covariance intersection filter," IEEE Intelligent transportation systems magazine, 5(2), pp.33-44, 2013.

[24] Karnadi, F.K., Mo, Z.H. and Lan, K.C. "Rapid generation of realistic mobility models for VANET," In Proceedings of the Wireless Communications and Networking Conference, WCNC-IEEE, pp. 25062511, 2007.

[25] "ArcGIS: editor for Open Street Map" [online] Available: https://www.arcgis.com/home/item.html?id=16970017f81349548d0a9eea d0ebba39

[26] Sheet, D.K., Kaiwartya, O., Abdullah, A.H., Cao, Y., Hassan, A.N. and Kumar, S., Location information verification using transferable belief model for geographic routing in vehicular ad hoc networks. IET Intelligent Transport Systems, 2016. (in press) [online published] 


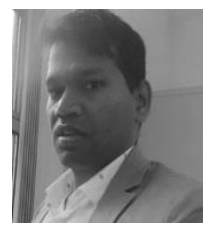

Omprakash Kaiwartya (M'15) received his $\mathrm{Ph} . \mathrm{D}$. degree in Computer Science from School of Computer and Systems Sciences, Jawaharlal Nehru University, New Delhi, India in 2015. He is currently a Research Associate at the Department of Computer and Information Sciences, Northumbria University, UK. He was Postdoctoral Research Fellow at the Faculty of Computing, Universiti Teknologi Malaysia, Johor Bahru, Malaysia. His research interests focus on Internet of connected Vehicles, Electronic Vehicles Charging Management, and IoT use cases in Sensor Networks.

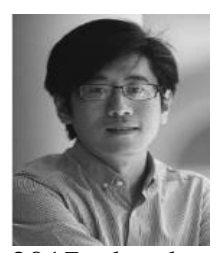

Yue Cao (M'16) received his $\mathrm{PhD}$ degree from the Institute for Communication Systems (ICS) formerly known as Centre for Communication Systems Research, at University of Surrey, Guildford, UK in 2013 and further as Research Fellow. Since August 2017, he has been the Senior Lecturer (previously was Lecturer since October 2016) in Department of Computer and Information Sciences, at Northumbria University, Newcastle upon Tyne, UK. His research interests focus on Delay/Disruption Tolerant Networks, E-Mobility, Autonomous Valet Parking, QoS/QoE in 5G. He is a reviewer of several IEEE journals and Associate Editor of IEEE Access.

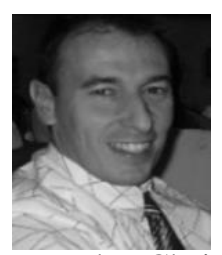

Jaime Lloret (M'07, SM'10) received his M.Sc. in Physics in 1997, his M.Sc. in electronic Engineering in 2003 and his Ph.D. in telecommunication engineering in 2006 . He is currently Associate Professor at the Universitat Politecnica de Valencia, Spain. He was the Chair of the Internet Technical Committee (IEEE Communications Society and Internet society) and Working Group of the Standard IEEE 1907.1. He has authored 22 book chapters and has more than 340 research papers published in national and international conferences, international journals (more than 110 with ISI). He has been the co-editor of 38 conference proceedings and guest editor of several international books and journals. He is editor-in-chief of the Ad Hoc and Sensor Wireless Networks, and associate editor of 46 international journals (16 ISI). He has been general chair of 28 International workshops and conferences.

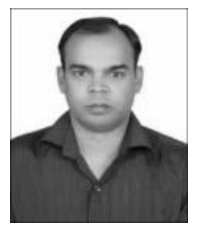

Sushil Kumar (M'14) received his Ph.D. degree in Computer Science from School of Computer and Systems Sciences, Jawaharlal Nehru University, New Delhi, India in 2014. He is currently an Assistant Professor at the Jawaharlal Nehru University, India. His research interest includes Vehicular Ad-hoc Networks, and Wireless Sensor Networks, and Internet of Things.

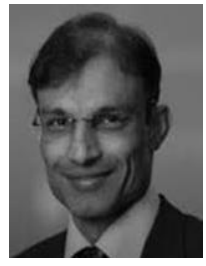

Nauman Aslam (M) received his Ph.D. in engineering mathematics from Dalhousie University, Canada, in 2008. He is currently a Reader (Associate Professor) in the Department of Computer and Information Sciences, Northumbria University, UK. He was an assistant professor at Dalhousie University from 2008 to 2011. He has extensive research experience in wireless ad-hoc and sensor networks.

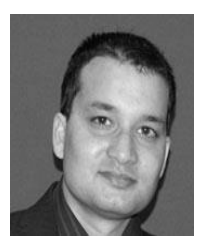

Rupak Kharel (M'17) received his Ph.D. degree in Computer Science from the Department of Computer and Information Sciences, Northumbria University, UK in 2011. $\mathrm{He}$ is currently a Senior Lecturer at the School of Engineering, Manchester Metropolitan University, UK. His research interests focus on Cyber Security oriented technical advancements for Internet of Things, Wireless Sensor Networks, and Industry 4.0.

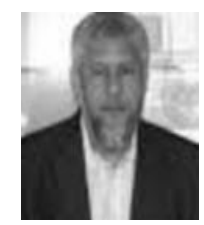

Abdul Hanan Abdullah (M'15) received his Ph.D. degree from Aston University in Birmingham, United Kingdom in 1995. He is currently a Professor and head of Pervasive Computing Research Group at Faculty of Computing, Universiti Teknologi Malaysia, Johor Bahru, Malaysia. His research interest includes Wireless Sensor Networks, Vehicular Adhoc Networks, and Next Generation Networks.

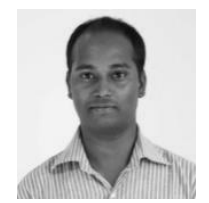

Rajiv Ratn (M'16) Shah received his Ph.D. from School of Computing, National University of Singapore (NUS) in 2016. He is a Postdoctoral Research Fellow at the School of Information Systems, Singapore Management University (SMU) in 2017. He is currently an Assistant Professor at the Indian Institute of Information Technology, Delhi (IIIT-D). His main research interests focus on multimodal analysis of user-generated content and locationbased services in support of social media applications, multimedia analysis and retrieval. 